\title{
Direct withdrawal of a knife in the lumbar spinal canal in a patient without neurological deficit: case report and review of the literature
}

\author{
Francisco Ismael Villarreal-García (10 ${ }^{1}$ • Pedro Martin Reyes-Fernández ${ }^{1}$ Oscar Armando Martínez-Gutiérrez ${ }^{1}$ • \\ Víctor Manuel Peña-Martínez ${ }^{1} \cdot$ Rodolfo Morales-Ávalos $^{1}$
}

Received: 25 February 2018 / Revised: 11 May 2018 / Accepted: 13 May 2018

(C) International Spinal Cord Society 2018

\begin{abstract}
Introduction Stab-wound injuries to the spinal cord are rare and currently, do not have a well-established management in the literature despite its strong association with neurological injury.

Case presentation We report a case of a patient with a stab-wound injury with a knife in the lumbar region, who underwent direct removal in the operating room without surgical exploration. Upon admission, the patient had no neurological deficit. The X-rays and CT scan before removal showed the involvement of $>50 \%$ of the intramedullary canal at L2. The removal was performed in the operating room with a surgical team available and ready in case there was neurological deterioration during the procedure.

Discussion We concluded that any neurologic deficit in a patient with a stab-wound injury in the spine must mandate surgical exploration and in patients without neurological deficit, direct removal of the stabbing object is a safe and effective method without adding the risks of a surgical exploration.
\end{abstract}

\section{Introduction}

The annual incidence of traumatic spinal cord injury worldwide is estimated at 35 patients per million population. The average age at the time of the injury is 32 years and the male to female ratio is 4:1. Motor vehicle and motorcycle accidents are the leading cause of traumatic spinal cord injuries, representing $>35 \%$ of spinal cord injuries per year. About $15 \%$ of traumatic spinal cord injuries are the result of violent encounters, often with firearms and blades or edged weapons and $9 \%$ are secondary to athletic activities, such as contact sports [1].

Stab-wound injuries in the spine are rare in developed countries, but continue to be a source of traumatic cord injury in developing countries. Due to the low incidence of these lesions in developed countries, its approach is not well established yet [2].

\section{Francisco Ismael Villarreal-García}

fco.villarrealgarcia@gmail.com

1 Servicio de Ortopedia y Traumatología, Hospital Universitario "Dr. José Eleuterio González", Universidad Autónoma de Nuevo León (U.A.N.L.), Monterrey, NL, Mexico
The currently reported management of patients with stabwound injuries in the spine and a neurologic deficit is surgical exploration, decompression, and removal of the foreign material in the case of retention in the spinal canal [37]. Surgical exploration in the management of patients without a neurological deficit is controversial due to the risk of injury during the procedure.

We report a case of a patient admitted to the Emergency Department with a stab-wound injury with a knife in the lumbar region and the approach and treatment established.

\section{Case presentation}

A 20-year-old male patient was admitted to the Emergency Department with a stab-wound injury with a knife in the lumbar region after having participated in an armed robbery 1-h prior admission (Fig. 1). On admission, the patient was hemodynamically stable and with 15 points in the Glasgow Coma Scale. While performing the initial assessment, we realized that the patient did not present any neurological deficit (the patient had normal sensory function and a full range of motion against normal resistance in the lower extremities, the rectal tone was normal and voluntary anal contraction was present). 
Ap and Lateral radiographs (Fig. 2) and a CT-Scan (Fig. 3 ) of the spine were requested to assess the position and direction of the knife, showing a midline trajectory in the lumbar spine passing through the inter-laminar space pointed towards the superior endplate of L2 involving $>50 \%$ of the intramedullary canal.

We performed direct withdrawal of the retained knife in the operating room under conscious sedation with a surgical team available and ready to perform a surgical exploration in case there was neurological deterioration, hemorrhage or

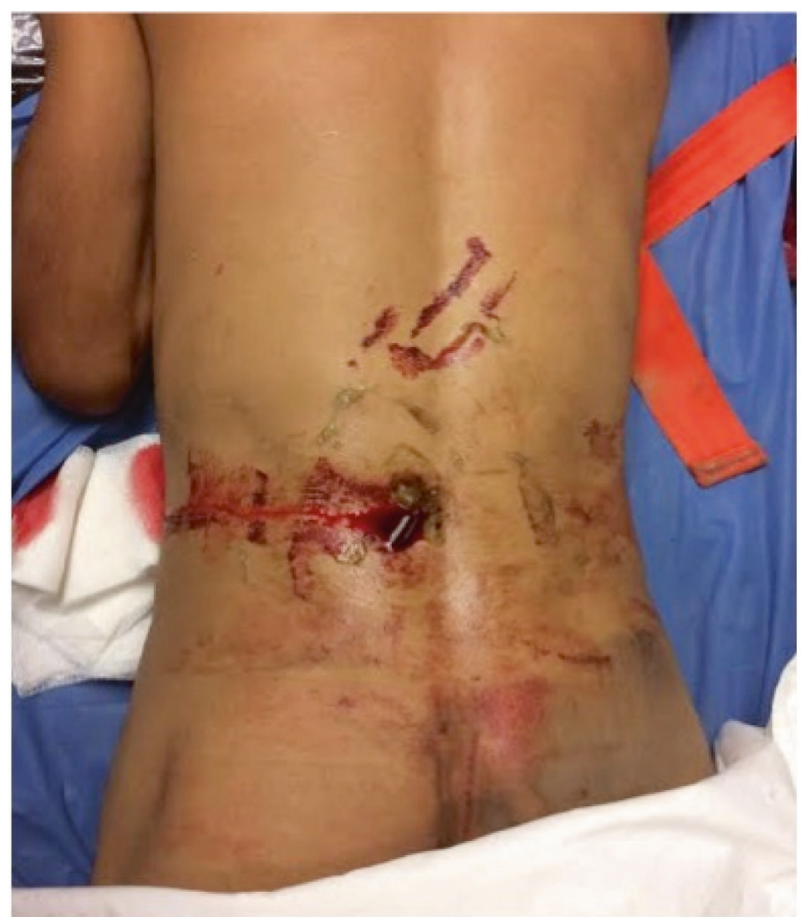

Fig. 1 Clinical image of the patient at admission in the Emergency Department

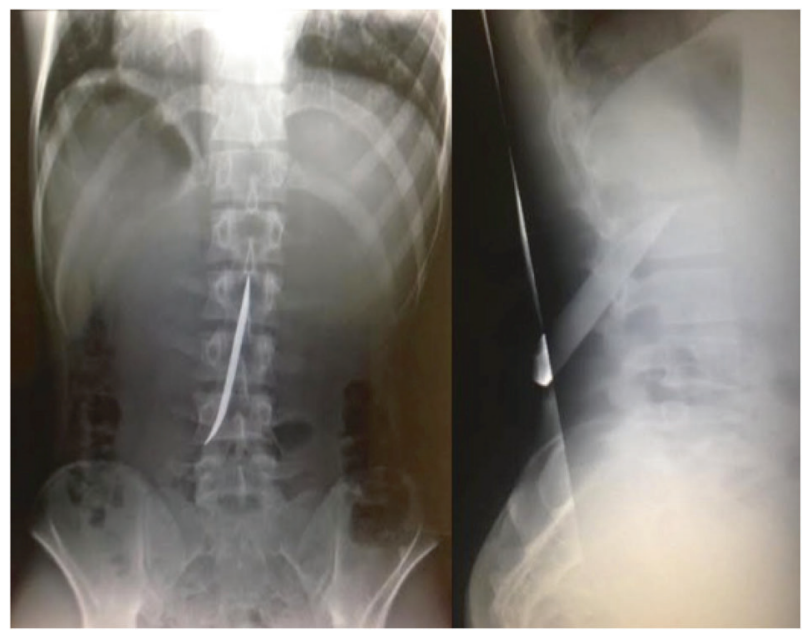

Fig. 2 AP and lateral radiograph showing the knife in L2 leakage of cerebrospinal fluid (CSF) during or after the procedure.

With the patient lying prone, the knife was retrieved from the wound applying gentle traction, paying attention to the presence of hemorrhage or leakage of cerebrospinal fluid (CSF) during or after the procedure. After the removal, radiographs were taken to rule out the presence of retained material in the spinal canal (Fig. 4). Irrigation and debridement of the wound with saline were performed and the wound was closed with simple sutures. Postoperative antibiotics were used and no steroids were administered. The patient had a normal neurological examination after the withdrawal.

The patient was kept under observation for three days without presenting any complication. A neurological examination was performed prior to discharge and the

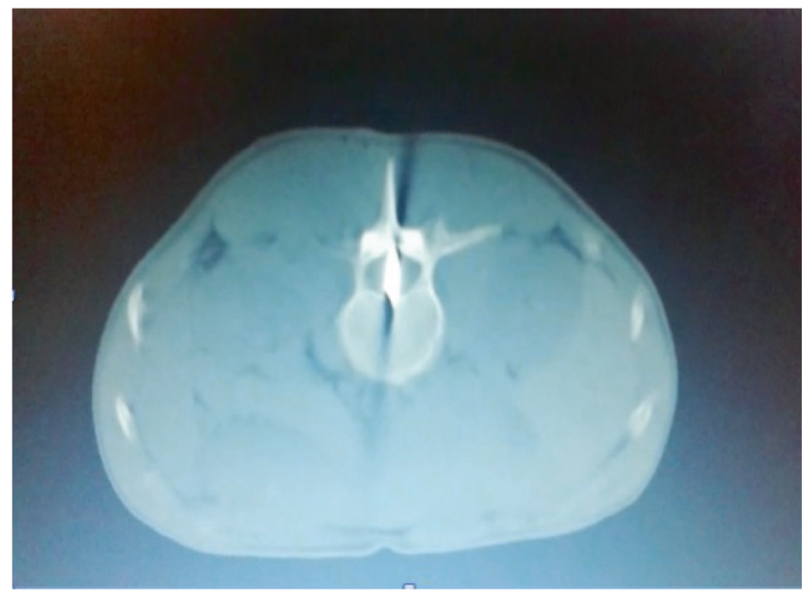

Fig. 3 Axial view of the CT scan showing the spinal canal involvement

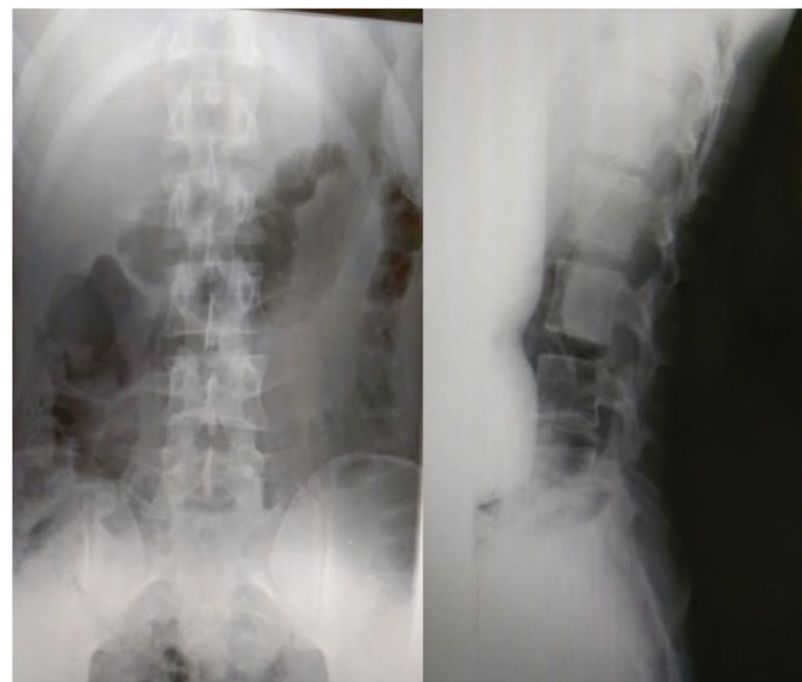

Fig. 4 AP and Lateral radiograph showing no retained material in the canal 
patient continued without any neurological sequel or pain and was able to walk without immobilization of the spine.

To our knowledge, this is the only case reported of a patient with a stab-wound injury in the spine by a foreign object (knife) with involvement of $>50 \%$ of the intramedullary canal presenting with a normal neurologic examination that was treated with direct removal of the knife and continued without neurologic deficit in the follow-up.

\section{Discussion}

The approach of the patient with penetrating trauma to the spine with and without neurological compromise must follow the ATLS (Advanced Trauma Life Support) protocol to rule out injuries that could put the patient's life at risk. Once the patient has been stabilized, it is important to perform a full neurological assessment before the withdrawal of the knife. It is necessary that the object must not be removed until radiographic studies are available to assess the position and possible compromised structures.

Stab-wound injuries to the spine are not common, but they are associated with a high percentage of neurological deficit. [4, 8].

Surgical exploration is mandatory in a patient with a neurological deficit at admission or deterioration while performing the withdrawal $[3,4]$. Peacock et al. in a review of 450 stab-wound spine injuries established indications for surgical exploration after withdrawal: leakage of cerebrospinal fluid (CSF), radiological signs of cord compression and retention of a foreign body in the medullary canal [2].

It should be emphasized that surgical exploration is not a harmless procedure and adds risks to the patient $[9,10]$ such as vascular injury, infection and the increase of the secondary damage to the previously established spinal cord injury. The benefits of the surgical procedure should be assessed against the risks of the patient [11].

Reported late complications such as myelopathy [12], intramedullary abscess, neurological deficit and spinal instability have been reported due to penetrating injuries in the spine [13, 14], none of them present in our patient prior discharge.

In his follow-up 3 weeks after discharge, the patient continued without any neurological deficit, the wound was closed and the patient returned to a fully functional level.
We concluded that any neurologic deficit in a patient with a stab-wound injury in the spine must mandate surgical exploration and in a patient without neurological deficit, direct removal of the stabbing object is a safe and effective method without adding the risks of a surgical exploration.

\section{Compliance with ethical standards}

Conflict of interest All the authors declare that they have no conflict of interest.

Informed consent ${ }^{*}$ The patient was asked for his written consent for the publication of his clinical case, mentioning that no personal information will be revealed.

\section{References}

1. Spinal cord injury facts and figures at a glance. National Spinal Cord Injury Statistical Center. https://www.nscisc.uab.edu/. Accessed 09 Jul 2014.

2. Peacock WJ, Shrosbree RD, Key AG. A review of 450 stab wounds of the spinal cord. S Afr Med J. 1977;51:961-4.

3. Thakur RC, Khosla VK, Kak VK. Non-missile penetrating injuries of the spine. Acta Neurochir. 1991;113:144-8.

4. Shahlaie K, Chang DJ, Anderson JT. Nonmissile penetrating spinal injury. Case report and review of the literature. J Neurosurg Spine. 2006;4:400-8.

5. Doğan S, Kocaeli H, Taşkapilioğlu MO, Bekar A. Stab injury of the thoracic spinal cord: case report. Turk Neurosurg. 2008;18:298-301.

6. Manzone P, Domenech V, Forlino D. Stab injury of the spinal cord surgically treated. J Spinal Disord. 2001;14:264-7.

7. Gulamhuseinwala N, Terris J. Evolving presentation of spinal canal penetrating injury. Injury. 2004;35:948-9.

8. Van den Berg ME, Castellote JM, Mahillo- Fernandez I, de PedroCuesta J. Incidence of spinal cord injury worldwide: a systematic review. Neuroepidemiology. 2010;34:184-92.

9. Velmahos GC, Degiannis E, Hart K, Souter I, Saadia R. Changing profiles in spinal cord injuries and risk factors influencing recovery after penetrating injuries. J Trauma. 1995;38:334-7.

10. Kossmann T, Trease L, Freedman I, Malham G. Damage control surgery for spine trauma. Injury. 2004;35:661-70.

11. Xia X, Zhang F, Lu F, Jiang J, Wang L, Ma X. Stab wound with lodged knife tip causing spinal cord and vertebral artery injuries: case report and literature review. Spine. 2012;37:931-4.

12. Sakar M, Dogrul R, Niftaliyev S, Bayri Y, Dagcınar A. Direct withdrawal of a knife lodged in the thoracic spinal canal in a patient with normal neurologic examination: is it safe? Spinal Cord Ser Cases. 2016;2:16009.

13. Jones FD, Woosley RE. Delayed myelopathy secondary to retained intraspinal metallic fragment: Case report. J Neurosurg. 1981;55:979-82.

14. Wright RL. Intramedullary spinal cord abscess. Report of a case secondary to stab wound with good recovery following operation. J Neurosurg. 1965;23:208-10. 